\title{
THE TWO COMPONENTS OF THE GRASP REFLEX AFTER ABLATION OF FRONTAL CORTEX IN ,MONKEYS*
}

BY

\section{GEOFFREY RUSHWORTH $\dagger$ and D. DENNY-BROWN}

\author{
From the Neurological Unit, Boston City Hospital, Boston, Mass., U.S.A., and the Department of Neurology, \\ Harvard Medical School
}

A grasp reflex was shown by Richter and Hines (1932) to result from experimental lesions in the pre-motor region (which they identified with area six of Brodmann) of the frontal lobes in adult monkeys. Reflex grasping in the monkey was later studied in more detail by Fulton and his associates in an effort to relate its occurrence to ablation of specific frontal areas and to analyse its nature at the periphery (Bieber and Fulton, 1933, 1938; Kennard and Fulton, 1933; Fulton, 1934). Since Janischewsky (1909) first described reflex hand grasping in a patient with organic brain disease, it has been the subject of many reports and several studies. Walshe and Robertson (1933) and Walshe and Hunt (1936) regarded the human grasp reflex as a purely proprioceptive reflex being devoid of visual or tactile elements, and a similar view of the simian grasp reflex was reached by Bieber and Fulton (1938). Seyffarth and Denny-Brown (1948) came to different conclusions for the human grasp reflex when they found that the adequate stimulus for it was a distally moving stimulus to certain areas of the palm. They were able to dissociate this triggering effect from the proprioceptive element. An essential sensory input for the reflex was derived from the skin and subcutaneous tissue of the palm, and this sensitized a secondary proprioceptive component when the stimulus stretched the long finger flexors. This secondary component reinforced and maintained the closing phase of the grasp initiated by palmar stimulation.

The large literature on reflex grasping shows many apparent discrepancies of observation and interpretation which are doubtless due to the confusion of terminology, lack of clear definition, and the failure to differentiate three distinct grasping automatisms (Seyffarth and Denny-Brown, 1948). In particular the lack of information regarding fixation

\footnotetext{
* Assisted by a grant from the Harrington Fund.

+On leave of absence from the University Laboratory of Physiology, Oxford. Present address: Neurological Research Unit, The Churchill Hospital, Oxford.
}

of the upper limb during application of the stimulus to the palm leads one to suppose that shoulder traction had not been recognized as a potent stimulus, of itself, of reflex closure of the fingers (the traction response).

In this paper we shall refer to the grasp reflex as the finger flexion, with thumb adduction that occurs in response to a distally moving pressure contact applied to the palm preceding traction on the finger flexors, with the shoulder, arm, and forearm fixed. It is regularly present after frontal lobe lesions in the monkey. It is to be compared with the "instinctive grasp reaction" which is the response to a light stationary or moving tactile stimulus on any part of the hand and it consists of orientation of the hand into a position necessary for finger-thumb palpation and final grasping of the object. This reaction, which is independent of vision, is occasionally found in the normal monkey, though the more usual response to a light tactile stimulus to the hand is one of slight withdrawal (avoiding). The instinctive grasp reaction is released by frontal lobe lesions in the monkey. Finally, the traction response has to be clearly differentiated from the other two grasping automatisms. Traction response is reflex finger flexion and thumb adduction resulting from abduction and flexion or traction on the shoulder, and it can also reinforce the grasping automatisms outlined above. It is a synergistic effect of stretching the spastic muscles at the shoulder and it is present therefore in the thalamic monkey as Bieber and Fulton (1938) described. It is unaffected by deafferentation of the hand and arm, but not the shoulder (Twitchell, 1954) and is modified, as are stretch reflexes, by head and neck turning. Though it is not a true grasp reflex (as defined above), an animal rendered spastic experimentally and showing the traction response can learn to use it for a hooking type of grasping such as hanging from a bar. Later it may learn to project the arm to initiate the traction response of flexion of the fingers which can then be hooked around an object. The subsequent slight 
stretching of the spastic finger flexors by the object may then result in closing the fingers through a facilitated stretch reflex and so lead to a fixed resistance to opening of the hand. It is therefore necessary to distinguish the purely passive traction response from grasping as a positive act. The present experiments were designed to clarify the grasp reflex of monkeys after frontal lobe ablation and to demonstrate the part played by the efferent muscle spindle innervation (gamma motor fibres) in this reflex.

\section{Methods}

Monkeys ( $M$. cyamologous) weighing between 2,200 and 3,000 g. were employed in this study. Their behaviour was watched for several days before operation and they were examined neurologically while strapped in a small chair designed to leave the limbs free while restraining the body. Under sterile conditions and nembutal anaesthesia (administered by the intraperitoneal route), the skull was opened by turning a left-sided bone flap. The central sulcus and the anatomical features of areas 4,6 , and 8 of Brodmann were identified. The left frontal pole was amputated, and areas 8 and 6 removed by subpial suction through small holes in the pia-arachnoid. The pia collapsed over the ablated areas. Much attention was given to the orbital surface and the cingulate (area 24) gyrus. The former was removed as far back as possible and the cingulate to the level of the medial limit of the central sulcus. After haemostasis had been secured, the dura was carefully repaired, the bone flap replaced and fixed by sutures in the galea. The muscles were closed in layers and the skin re-approximated by continuous subcutaneous silk suture.

By the next day the animals were usually quite active and at the end of a week the wound had healed sufficiently to allow of catching the animal in a net and strapping it into a special chair for neurological examination and experiment. The chair was provided with a wooden arm projecting at right angles to the chair-back and the animal's right forearm was fixed to this by strips of "elastoplast" around the elbow and wrist. A light rubber band was fixed to the finger tips and attached to a Grass strain-gauge myograph with sufficient tension to keep the fingers just extended. A small concentric needle electrode was placed within the flexor muscles of the fingers and lightly fixed there. The potentials picked up by this and the output of the strain-gauge were suitably amplified and displayed on a cathode ray tube which was photographed on moving paper by means of a Grass camera. The stimulus for the grasp reflex was provided by a small insulated metal strip fitted with electrical contacts. The strip was moved with moderate pressure across the palm beginning proximally and the initial contact with the palm was signalled automatically as was the increased resistance to movement as the stimulator encountered the flexing fingers. The grasp reflex so elicited was studied before and after injection of $1 \%$ procaine around the ulnar and median nerves at the wrist, and in separate experiments small quantities of procaine were injected into the flexor muscles of the fingers them-
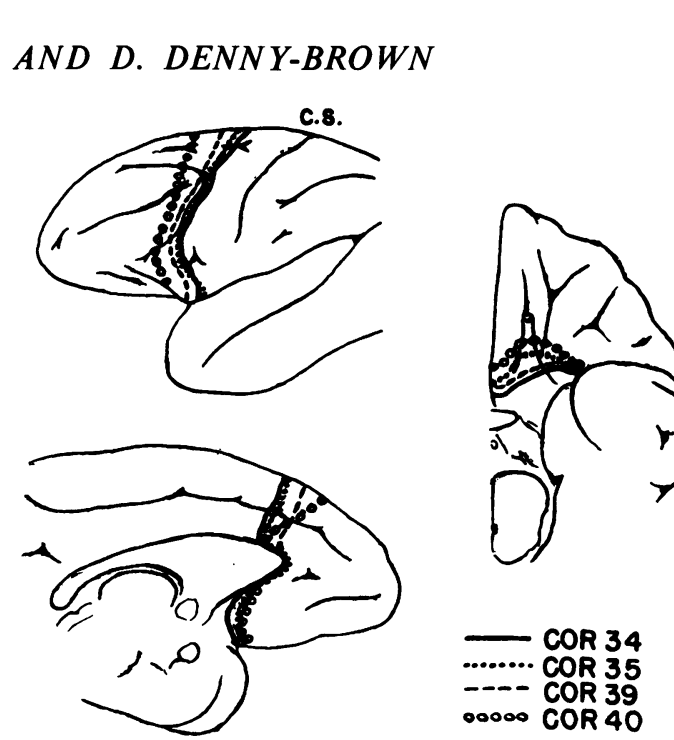

FIG. 1.-The extent of ablation of the left frontal lobe in thes four monkeys described in the text, charted from the necropers specimens. Where the ablation was bilateral it was approximatefr. symmetrical. In animals COR 34 and COR 35 the entire corte in front of the precentral sulcus was removed, whereas in animats COR 39 and COR 40 the precentral gyrus remained. C.S. is the central sulcus.

selves. Finger jerks, response to pin prick in the paim effects of turning the head and neck, and spontane struggling movements could be recorded.

About six weeks after the first operation, the procedur was repeated in the right side of the head and the gasp reflex studied in both hands. The behaviour of animals after frontal lobe lesions is to be reported gl later date. The extent of the ablations in the four animals discussed in this paper is shown in Fig. 1, and 8 drawn from the necropsy specimens which had bee fixed in situ.

\section{Results}

Within one week of operation a grasp reflex was regularly elicitable from the palm contralateral to the cortical lesion and more facile avoiding responses from the homolateral limbs ("transcortical release" When the animal was strapped into the experimental chair and its forearm fixed to the chair arm, a grasp reflex was still elicitable, though greater pressure ô the moving object was now necessary to elicit the reflex every time. It was also necessary to see that the dorsum of the fingers and hand did not make contact with any part of the experimental chair because tactile stimuli to the dorsum of the hand are powerful inhibitors of the grasp reflex. Neither the attaching of the fingers to a myograph through light rubber band nor the insertion of a small covo axial electrode into the finger flexors had any significant effect on the grasp reflex.

By slowly moving the stimulating object across the palm on to the fingers using moderate pressure? 

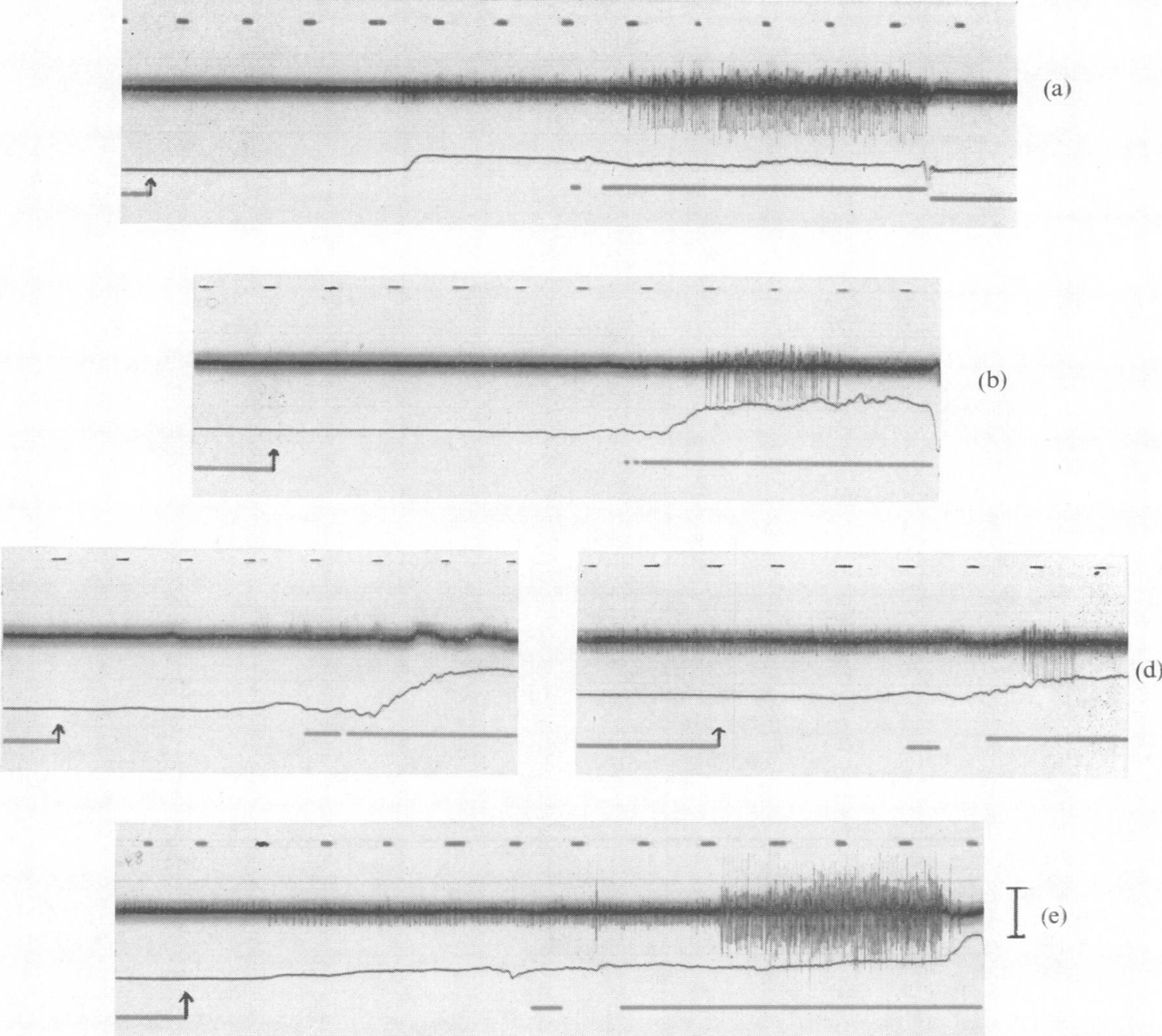

FIG. 2.-(a) The two components of the grasp reflex in the right hand of an animal (COR 35) with ablation of the left frontal lobe including areas 6, 8, and the cingulate gyrus. Electromyogram from finger flexors and myograph attached to finger. Stimulus applied to palm at the arrow; and at the continuous line during stretching of the finger. Time above in 1 seconds; (b) ten minutes after procaine infiltration around the ulnar and median nerves at the wrist (a small stretch reflex only remains); (c) twenty minutes later neither grasp reflex nor stretch reflex; (d) the same, with head turned strongly to the right when a small stretch reflex can be elicited in the finger flexors; (e) fortyeight minutes after the procaine injections, the grasp reflex begins to return. Voltage calibration in this and subsequent figures $100 \mu \mathrm{v}$.

a constant type of response in the finger flexors was invariably seen. Contact with the skin of the most proximal palm had at first no effect, but as the stimulus moved more distally rapid recruitment of small motor units was seen in the electromyogram and the fingers flexed slightly (Fig. 2a), particularly at the interphalangeal joints. They were in this state of partial flexion when the stimulus reached them and caused slight stretching of the finger flexors producing an outburst of large action potentials in the electromyogram and closing of the fingers on to the object. In some animals tonic grasping lasted for several minutes even when the stimulus was removed from the grasp, but nevertheless the outburst of large action potentials usually ceased when the stimulus no longer stretched the finger flexors. In this way, the two components, first cutaneous then proprioceptive, which provide the adequate stimulus for the grasp reflex, are demonstrable. Their relationship to each other may be analysed by 

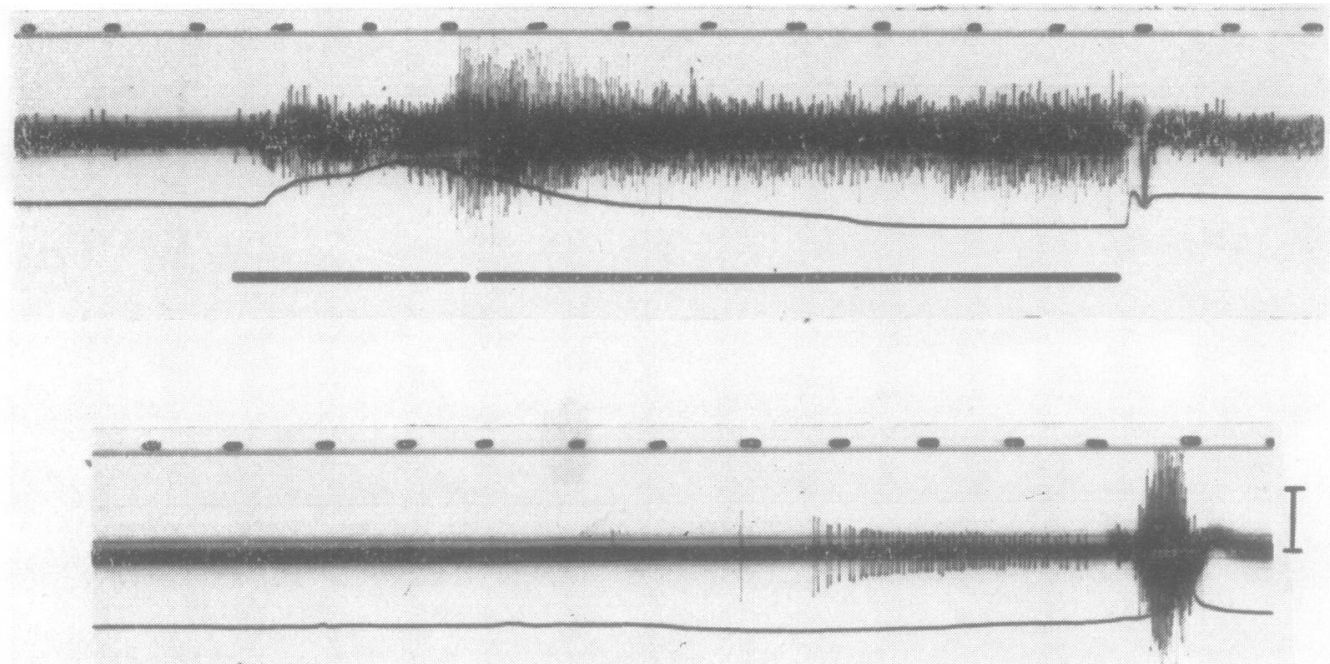

FIG. 3.-Electromyogram from another animal (COR 39) showing right grasp reflex. Palmar stimulus applied at beginning of first continuous

(a) Before procaine; (b) six minutes after procaine infiltration around ulnar and median nerves at the wrist. A stretch reflex elicited ợ $\$$

on extreme stretching of the fingers. The record ends with a brief tetanic finger flexion of a spontaneous voluntary struggle by the anirial

injecting $1 \%$ procaine around both the ulnar and median nerves at the wrist so as to block the purely palmar stimuli.

Fig. $2 b$ shows the state of affairs 10 minutes after procaine infiltration at the wrist. There is now no electromyographic response nor do the fingers flex when the object moves distally in the palm. Initially, stretch of the finger flexors has no effect but with greater stretch (as the stimulus reaches the middle and distal phalanges) a brief stretch reflex is seen. The fingers then exhibit a very mild resistance to passive stretch, but no grasping results from this proprioceptive stimulation. Twenty minutes later not even a small stretch reflex is elicitable (Fig. 2c), but if the head is turned strongly to the right (Fig. 2d) a little background activity is now seen in the electromyogram and a small stretch reflex from the finger flexors, but no grasp reflex. Forty-eight minutes after the procaine injection, the grasp reflex begins to return (Fig. 2e) with the small finger flexion in response to the cutaneous stimulation and the final tetanic closing phase being associated with stretching of the finger flexors.

In another animal (Fig. 3a), which showed a well developed tonic grasp reflex, the injection of procaine around ulnar and median nerves at the palm led to complete disappearance of the grasp reflex within six minutes of injection (Fig. 3b) though a feeble stretch reflex (a single unit in the electromyogram) was still present. The animal could still (and often did) "voluntarily" and maximally flex the fingers, is shown by the outburst of action potentials in electromyogram at the end of the record shown Fig. 3b. When the procainized hand had lost gits grasp reflex it was still possible to obtain a graspo reflex in the foot of the same side. This wo alo indicate that the effect of procaine injected at the wrist on the grasp reflex of the hand is indeed a locap effect and not due to the systemic effects of procaines

In two of the monkeys a small strip of areas 6 anc 8 immediately adjoining area 4 was purposely spared Both these animals exhibited grasp reflexes but the tonic closure of the fingers was briefer than in the animals with more complete lesions. Fig. 4a shows such an example. Rapid recruitment of motor units is shown in the electromyogram in response to the cutaneous stimulation, and stretching of the fingers produces a relatively brief tetanic outburst whict persists only as long as the fingers are stretched? Seven minutes after injection of $1 \%$ procaine around ulnar and median nerves at the wrist (Fig. 4b) a vere little finger flexion occurs in response to the stimulus in the palm, but there is a stretch reflex which persist? only as long as the muscles are stretched. It was possible in this animal to note that an area of skit. overlying the heads of the second, third, and fourth metacarpals was particularly active in conditioning the stretch response in the finger flexors and this was still present 11 minutes after the procaines injection (Fig. 4c). By the seventeenth minute afte 
Fig. 4.-Animal with incomplete ablation of areas 6 and 8 (COR 40) left grasp reflex.

(a) Before procaine; large response to cutaneous stimulation, relatively little added to this by finger stretching; (b) seven minutes after procaine infiltration of ulnar and median nerves at the palm, no grasp reflex, a stretch reflex persists; (c) eleven minutes later. The skin over heads of second, third, and fourth metacarpals conditions an active stretch reflex in the finger flexors; (d) seventeen minutes later. No response to either cutaneous or proprioceptive stimuli.

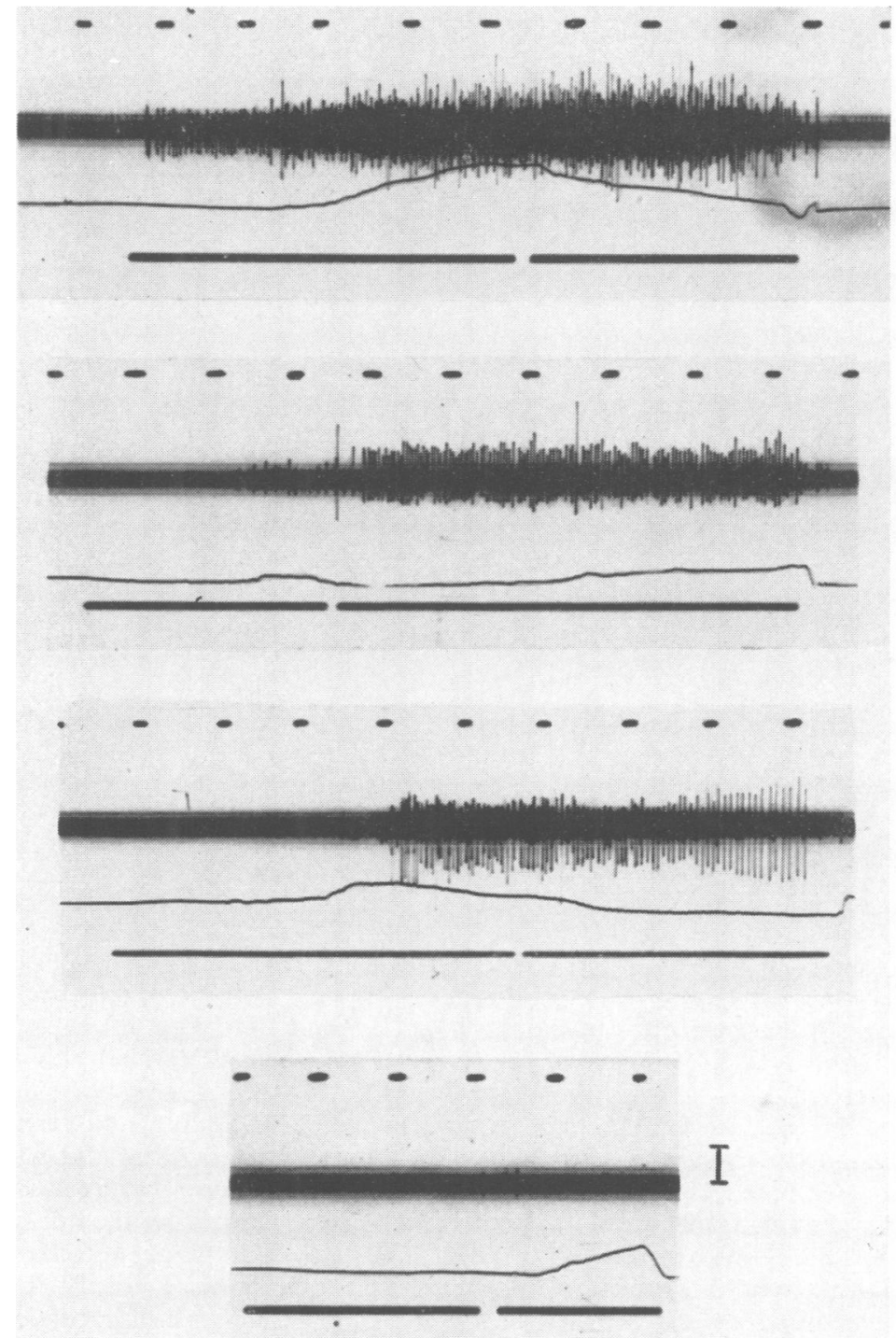

injection of procaine both the grasp reflex and the stretch reflex had disappeared (Fig. 4d) and the skin of the palm now appeared to be quite anaesthetic, i.e., the monkey did not respond to any form of finger or palmar stimulation.

These experiments demonstrate that the grasp reflex in the monkey is triggered off by cutaneous stimuli which condition the finger flexors in such a way that when the stimulus stretches these muscles then grasping occurs. Stretching the muscles alone without the initial cutaneous component fails to elicit the grasp reflex, and, indeed, if the cutaneous component is completely abolished by procaine then the stretch reflex of the finger flexors is absent.

In one animal showing a grasp reflex after ablation of the frontal lobe, including areas 6,8 , and cingulate, at a subsequent operation several weeks later, the ulnar nerve was divided at the wrist and a week later the median nerve. After division of the ulnar nerve a grasp reflex was obtainable only by stimulating the median distribution of the palm, and the fifth finger did not take part in the reflex and the fourth only partly. After the additional division of the median nerve, a grasp reflex could not be elicited even by a combination of finger stretching and head turning. There was, however, mild 


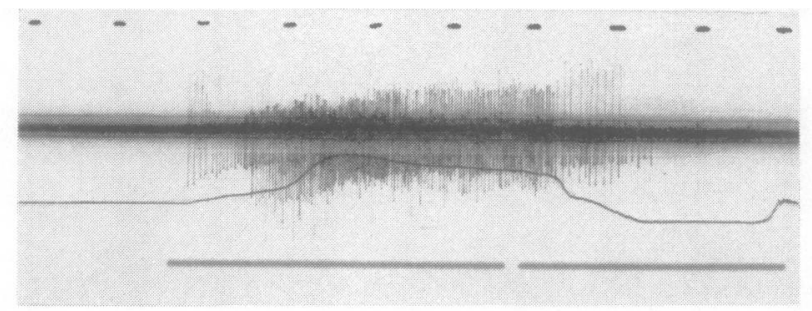

(b)

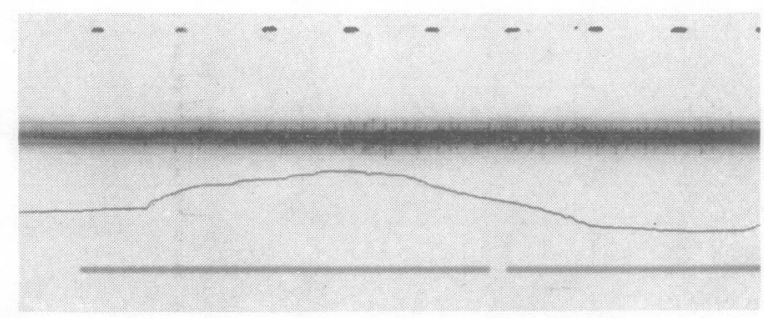

(c)

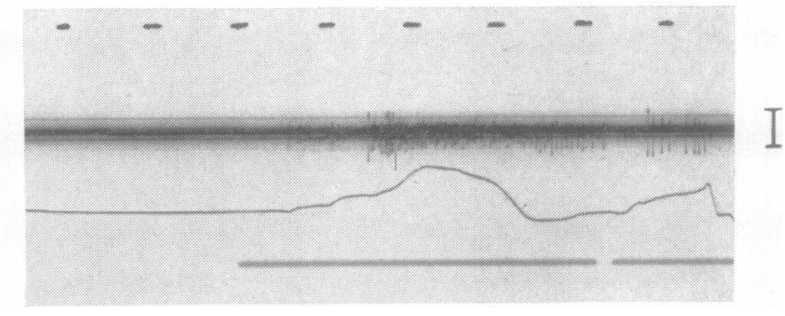

Fig. 5.-Cor 40 right hand (left areas 6 and $8 \stackrel{3}{=}$ spared).

(a) The grasp reflex with little added to the cutaneous response by the proprioceptive $\overrightarrow{\vec{S}}$ stimulus; (b) two minutes after injection of $0.2 \mathrm{ml}$. of $1 \%$ procaine into the flexor muscles? of the fingers. Very feeble flexion in response $\bar{\sigma}$ to cutaneous stimulation. Nothing added $\overline{\bar{N}}$ by finger stretching; (c) eleven minutes later, finger flexion begins but a very brief stretch $\odot$ reflex does not complete the act of grasping. resistance to passive stretch of the fingers, i.e., mild spasticity.

The response of a muscle to stretch depends not only on the excitability of its motoneurone pool but also on the sensitivity of its muscle spindles to stretch. Gamma motoneurones (fusi-motoneurones) in the spinal cord specifically innervate muscle spindles, and in this way their sensitivity can be controlled by the central nervous system. Stretch reflexes may be greatly exaggerated by the activity of gamma motor fibres, as for example, in decerebrate rigidity (Matthews and Rushworth, 1957a). It was thought possible that the cutaneous component of the adequate stimulus for the grasp reflex not only activated the motoneurone pool of the finger flexors causing partial finger flexion, but also activated the gamma motoneurones (fusi-motoneurones) of their muscle spindles, and thus conditioned the muscles to react tonically to stretching when the stimuli reached them. There is good evidence (Matthews and Rushworth, 1957b, 1958) that procaine tends to anaesthetize the gamma motor fibres of muscle spindles before either large motor fibres or large afferents and it was of interest therefore to observe the effects of injection of small quantities of procaine into the finger flexor muscles of monkeys aftêf frontal lobe ablation. In every case the grasp reflex disappeared, usually within one minute of injection, and reappeared about 15 to 20 minutes later. Spontaneous movements by the animal during the period of absent grasp reflex were rare, $\frac{\mathrm{O}}{\mathbb{Q}}$ but when they did occur they indicated, of course, $\stackrel{a}{\vec{a}}$ that large motor fibres were still functional. Fig. 5a음 shows a grasp reflex in an animal which exhibited rather a short tonic grasping phase after the stimulus reached the fingers and stretched them. Two minutes after injection of $0.2 \mathrm{ml}$. procaine into the flexor muscles of the fingers (Fig. 5b), the fingers were very? feebly flexed in response to the cutaneous stimulation and nothing was added to this by stretching the finger flexors. Eleven minutes after the procaineo injection (Fig. 5c) a small finger flexion occurs in response to the cutaneous stimulus and a very small음 stretch reflex when the fingers are stretched. This is the type of "desensitized" stretch reflex one would을 expect to see if the gamma motor fibres to then spindles of the injected muscles had been blocked. The result indicates that most, if not all, the prelimin- $N$ ary closing reaction as well as the proprioceptive $N$ component is mediated through the gamma system. 
Fig. 6.-Two animals with frontal lobe ablations sparing areas 6 and 8.

(a) COR 24. Heavy stimulus applied to palm at arrow. Very little recruitment of motor units, until stimulus reaches skin over metacarpal phalangeal joints when suddenly fingers flex followed at once by extension and silence in the electromyogram; (b) COR 40. Pin prick to the palm (signal below) during some activity of the finger flexors, brief grasp followed by avoiding of the fingers, prolonged after the second stimulus.

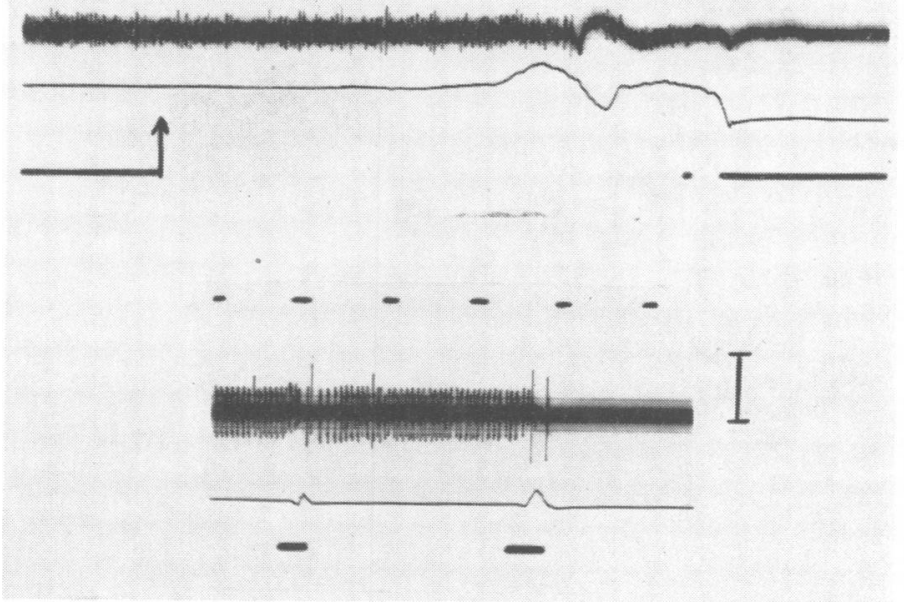

The poorly sustained tonic grasp of the animals with intact areas 6 and 8 is of some interest. It appears that excessive stretch on the fingers or excessive stimulus to the palm tends to inhibit grasping. In Fig. 6a a heavy stimulus was applied to the palm resulting in very little recruitment of motor units in the finger flexors and then suddenly a little flexion rapidly followed by extension of the fingers. Similarly in these animals pin prick to the palm caused a small finger flexion followed by extension, clearly seen if recordings were taken from the flexor muscles when there was some background activity (Fig. 6b). The usual response to pin prick in the palm of animals lacking completely areas 6 and 8 is one of finger flexion only (a brief grasp). It appears, therefore, that the sparing of areas 6 and 8 was responsible for the avoiding (extension) reactions in response to pin prick and heavy pressure. The avoiding reactions probably require the presence of areas 6,8 , and 24 for their full development (Denny-Brown, 1951).

\section{Discussion}

The grasp reflex after frontal lobe lesions in the monkey occurs as a result of a combination of cutaneous and proprioceptive stimulation. Of these, the cutaneous component is the essential initiator of the reflex and without this proprioceptive stimulation is ineffective. This is in complete agreement with the results of Seyffarth and Denny-Brown (1948) on the human grasp reflex. The opposite opinion of Bieber and Fulton (1938) that the grasp reflex in the monkey following frontal lobe lesions is a postural mechanism independent of cutaneous sensory influx is almost certainly due to the confusion between the "traction response" and the "grasp reflex". This may also account for the results of Walshe and Robertson (1933) and Walshe and Hunt (1936) for human grasping responses. Our own experiments clearly show that when palmar sensation is abolished in the monkey by either procaine infiltration at the wrist or by actual section of ulnar and median nerves, then the grasp reflex cannot be elicited.

Palmar stimulation by a distally moving object causes some initial finger flexion (at interphalangeal joints particularly), but the long finger flexors are rendered hypersensitive to stretch so that when the stimulating object stretches them the fingers will close on it. This overactive stretch reflex is almost certainly due to activation of the gamma motoneurones (fusi-motoneurones) which innervate the muscle spindles of the finger flexors causing their stretch receptors to become particularly sensitive. The local anaesthetic procaine, when placed around a muscular nerve, blocks gamma motor fibres in preference to either large motor fibres or large sensory fibres (Matthews and Rushworth, 1957b). The injection of very small quantities of procaine into the finger flexors rapidly abolished the grasp reflex, without necessarily having an effect on the power of contraction of those muscles. The integrity of gamma motor fibres to the muscle spindles of the finger flexors is shown to be necessary, not only for the final proprioceptive response of the grasp reflex, but also for the tactile facilitatory mechanism (closing phase) that prepares the finger flexors for stretch. The closing phase may thus be considered 
a gamma response of which the facilitatory (subliminal) aspects are much wider than the small finger flexion.

The grasp reflex may be demonstrated in cats with high spinal lesions (Denny-Brown, Rushworth, and Saenz-Arroyo, to be published) and its essential factors are therefore a spinal reflex employing simple segmental pathways. In the cat, also, section of ulnar and median nerves at the wrist abolishes the grasp reflex, but the digits now exhibit a mild resistance to stretch, i.e., they have become spastic (DennyBrown, Twitchell, and Saenz-Arroyo, 1949). It appears that sensory influx from the fore-paw has a mixed inhibitory and excitatory effect on the motoneurone pool of the finger flexors; in the circumstances, when reflex grasping is prominent, the proprioceptive reflexes are depressed (or rather, dominated) by the cutaneous afferent system until a specific moving tactile stimulus triggers their release. It can be demonstrated experimentally that the reflex effects of median nerve stimulation at the wrist is, indeed, a complex mixture of inhibition and excitation on limb muscles with a tendency to produce extension of the forelimb (Denny-Brown and Liddell, 1928).

In one monkey experiment when the ulnar and median nerves were separately sectioned at the wrist, the fingers at first showed no increased resistance to passive displacement until four or five days after the second operation when they exhibited very mild spasticity with increased finger jerks. An increased stretch reflex of the finger flexors was never seen after procaine infiltration at the wrist, and the question then arises as to this difference between the experiments with procaine and those with nerve section. In both respects, however, the outcome is complicated by the avoiding response which is difficult to eliminate entirely by cortical ablation, and has frequently been found to be the reason for the transitory failure of motor reactions after cortical ablations (Denny-Brown and Chambers, 1958). The avoiding response has a much wider reflexogenous area in the upper limb than the grasp response. The situation is less complex in the animal with hemisection of the spinal cord and is being further examined in this context. The excitatory properties of the cutaneous stimulus have already been described in detail, but we have not so far considered any inhibitory activity on the stretch reflex mechanism which could be arising distal to the wrist. Possibly proprioceptors near the joints of the digits may inhibit the stretch reflex of the finger flexors, and the large afferents of such sensory organs would be expected to be very resistant to procaine. Stretch reflexes then would not be released by procaine infiltration but would be expected from total nerve section at the wrist. However, such a mechanism remains, at the moment, $\underset{Q}{Z}$ purely speculative.

The persistence of avoiding reactions of the fingers (extension) in response to pin prick or excessive stretch in animals with lesions of the frontal lobe, $\square$ but sparing areas 6 and 8 , again confirms the importance of these areas in the avoiding reactions? of normal animals or animals lacking parietal lobes (Denny-Brown, 1951; Denny-Brown and Chambers,, 1958).

Summary
A grasp reflex can be elicited in the contralateral hand after frontal lobe ablations (including cingulate $\overline{\bar{c}}$ gyrus and areas 6 and 8 ) in monkeys.

The adequate stimulus for it is a distally moving object causing stimulation first of the palm and then ${ }^{c}$ of the fingers.

Local anaesthesia of the palm with procaine $\vec{F}$ abolishes the grasp reflex which cannot be elicited ${ }_{\sigma}^{\omega}$ by proprioceptive stimulation alone (stretching of the fingers).

The palmar cutaneous component of the stimulus conditions the finger flexors for the subsequent stretch stimulus.

This conditioning results in partial finger flexio especially at the interphalangeal joints (the closiris phase), and also an increased stretch response of the muscles through activation of the gamma mote neurones (fusi-motoneurones) to the muscle spindle?.

Nerve section at the wrist abolishes the gras reflex but the fingers then show very mild spasticit

Sparing of areas 6 and 8 allows some persistence of avoiding reactions in the hand to pin prick and excessive finger stretch and the grasp reflex is relatively poorly maintained.

One of us (G.R.) expresses his appreciation of a research travel grant from the Wellcome Trust and the $\overrightarrow{\vec{O}}$ National Spastics Society which made this work possible.

\section{REFERENCES}

Bieber, I., and Fulton, J. F. (1933). Amer. J. Physiol., 105, 7. $\overline{1}, \frac{1}{1938)}$. Arch. Neurol. Psychiat. (Chicago), 39, 433. Denny-Brown, D. Unpublished observation.

(1951). "The Frontal Lobes and Their Functions." Ch. 2 in Modern Trends in Neurology, p. 13-89, ed. by A. Feiling. Butterworth, London.

and Chambers, R. A. (1958). Res. Publ. Ass. nerv. ment. Dis., 36,35 .

- , and Liddell, E. G. T. (1928). J. Physiol. (Lond.), 65, 305

Denny-Brown, D., Rushworth, G., and Saenz-Arroyo, L. To be published.

Twitchell, T. E., and Saenz-Arroyo, L. (1949). Trans. Amer. neurol. Ass., 74, 108.

Fulton, J. F. (1934). Arch. Neurol. Psychiat. (Chicago), 31, 221.

Janischewsky, A. (1909). Rev, neurol., 17, 823.

Kennard, M. A., and Fulton, J. F. (1933). Brain, 56, 213. Kennard, M. A., and Fulton, J. F. (1933). Brain, 56, 213.
Matthews, P. B. C., and Rushworth, G. (1957a). J. Physiol. (Lond.),
135, 245. 135, 245. (1957b). Ibid., 135, 263.

,$--(1957 b) . \quad$ Ibid., 135, 263.

Richter, C. P., and Hines, M. (1932). Amer. J. Physiol., 101, 87.

Seyffarth, H., and Denny-Brown, D. (1948). Brain, 71, 109.

Twitchell, T. E. (1954). J. Neurophysiol., 17, 239.

Walshe, F. M. R., and Hunt, J. H. (1936). Brain, 59, 315

, and Robertson, E. G. (1933). Ibid., 56, 40. 